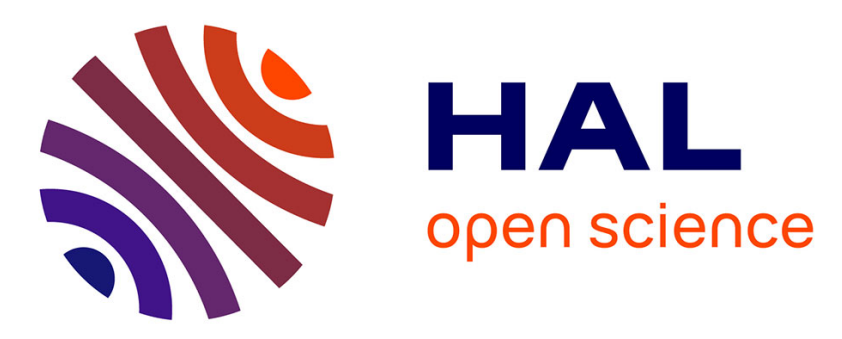

\title{
Impact of chirality on hydrogen-bonded supramolecular assemblies and photoconductivity of diketopyrrolopyrrole derivatives
}

Swann Militzer, Nozomi Nishimura, Nelson Ricardo Ávila-Rovelo, Wakana Matsuda, Duncan Schwaller, Philippe Mesini, Shu Seki, Amparo Ruiz

\author{
Carretero
}

\section{To cite this version:}

Swann Militzer, Nozomi Nishimura, Nelson Ricardo Ávila-Rovelo, Wakana Matsuda, Duncan Schwaller, et al.. Impact of chirality on hydrogen-bonded supramolecular assemblies and photoconductivity of diketopyrrolopyrrole derivatives. Chemistry - A European Journal, 2010, 10.1002/chem.202001540 . hal-02868137

\section{HAL Id: hal-02868137 https://hal.science/hal-02868137}

Submitted on 8 Jan 2021

HAL is a multi-disciplinary open access archive for the deposit and dissemination of scientific research documents, whether they are published or not. The documents may come from teaching and research institutions in France or abroad, or from public or private research centers.
L'archive ouverte pluridisciplinaire HAL, est destinée au dépôt et à la diffusion de documents scientifiques de niveau recherche, publiés ou non, émanant des établissements d'enseignement et de recherche français ou étrangers, des laboratoires publics ou privés. 


\title{
Impact of chirality on hydrogen-bonded supramolecular assemblies and photoconductivity of diketopyrrolopyrrole derivatives
}

\author{
Swann Militzer, ${ }^{[a]}$ Nozomi Nishimura, ${ }^{[b]}$ Nelson Ricardo Ávila-Rovelo,,${ }^{[a]}$ Wakana Matsuda, ${ }^{[b]}$ Duncan \\ Schwaller, ${ }^{[a]}$ Philippe J. Mésini, ${ }^{[a]}$ Shu Seki, ${ }^{[b]}$ and Amparo Ruiz-Carretero ${ }^{*[a]}$
}

[a] S. Militzer, N. R. Ávila-Rovelo, D. Schwaller, P. J. Mésini, A. Ruiz-Carretero

University of Strasbourg, Institute Charles Sadron, CNRS, UPR22

23 Rue du Loess, 67000 Strasbourg Cedex 2, France

E-mail: Amparo.ruiz@ics-cnrs.unistra.fr

[b] N. Nishimura, Dr. W. Matsuda, Prof. S. Seki

Department of Molecular Engineering, Graduate School of Engineering

Kyoto University

Nishikyo-ku, Kyoto 615-8510, Japan

Supporting information for this article is given via a link at the end of the document.

\begin{abstract}
Hydrogen bonds can efficiently guide the self-assembly of organic materials, being possible to tune the properties during the aggregation process. In the case of $\pi$-conjugated materials, several parameters such as temperature, concentration and solvent can be used to modify the aggregation state while tuning the optoelectronic properties. Chirality can be included within the impacting parameters due to the differences in molecular packing. Here we design and synthesize chiral and achiral thiophene-capped diketopyrrolopyrrole derivatives containing amide bonds with the aim to study the interplay between chiral assemblies and their stabilization via hydrogen-bonding Differences in aggregation properties were observed with spectroscopy and microscopy, and a contactless microwave-based technique was used to study their intrinsic charge carrier mobility. The positive role of hydrogen-bonding has been highlighted and the differences between chiral and achiral compounds have been elucidated.
\end{abstract}

\section{Introduction}

Conjugated materials, polymers[1] and small molecules,[2] have been extensively explored in the last decades due to their potential in the field of organic electronics.[3,4] Great progress has been reported in the fabrication of field-effect transistors (FETs),[5] light-emitting diodes (LEDs),[6] solar cells[7] and thermoelectric materials.[8] The optoelectronic properties of $\pi$-conjugated materials as well as their morphological features need to be carefully explored to achieve the best performance in the final devices. There have been global concerted efforts dedicated to the optimization of the optoelectronic properties, including the use of push-pul strategies,[9] the incorporation of heteroatoms[10] in the molecular structure or the extension of the conjugation length.[11] Regarding the structural properties of organic semiconductors, they can be controlled by rational design[12] or by applying different processing techniques, such as thermal or vapor annealing,[13,14] alignment techniques,[15] vacuum deposition[16] and the use of additives.[17] In this sense, the application of supramolecular chemistry strategies, in which noncovalent interactions form highly organized semiconducting structures could be very beneficial.[18] Directional noncovalent interactions, such as hydrogen bonds $(\mathrm{H}$ bonds), can be introduced in the semiconducting segments and guide the self-assembly towards different supramolecular structures with different optoelectronic properties.[19-21] This is due to the high sensitivity of $\mathrm{H}$-bonds to external stimuli and their environment. Chirality can impact the self-assembly properties as well, and in the case of semiconductors their optoelectronic properties.[22,23]. The chirality is amplified when the molecular chirality is transferred to the supramolecular structures, which makes new properties emerge. Supramolecular chirality has been explored in organic semiconductors using $\pi-\pi$ stacking and $\mathrm{H}$-bonding mainly.[22,24-27] In this work, we explore hydrogen-bonded diketopyrrolopyrrole (DPP) derivatives containing chiral and achiral centers. DPP[28] dyes are among the most explored semiconductors in the organic electronics field since they are easily functionalized in different positions and they have shown excellent properties in different types of devices.[29-31] Aromatic moieties are usually attached to the DPP core to tune the optoelectronic properties and they are normally modified with electron-donating or withdrawing groups.[32] The incorporation of $\mathrm{H}$-bonds also impact the self-assembly and optoelectronic properties of DPP derivatives without extending the conjugation length.[19] Chirality has been introduced in DPP derivatives mainly by linking them to chiral alkyl tails, usually 2 ethylhexyl[33-36], to helicene molecules,[37] or adding myrtenal derivatives.[38] Regarding chiral and H-bonded DPP derivatives, Brauchsweig[39-43] reported examples where DPPs with chiral side chains were attached to a diaminopyridine (DAP) H-bonding unit to study supramolecular donor-acceptor systems. Here we report three DPP derivatives containing amide groups pending from the lactam rings: a linear achiral molecule and two chiral ones. A combination of variable temperature UV-Vis, Fourier transform infrared (FTIR) and circular dichroism (CD) spectroscopy has been used to understand the different assembly processes in solution and on thin films. Their morphology in different solvents was analyzed by transmission electron microscopy (TEM). The impact of $\mathrm{H}$ - 
bonding and chirality on the charge transport properties was studied by flash-photolysis time-resolved microwave conductivity (FPTRMC), indicating the positive role of $\mathrm{H}$-bonding on electric conductivity and showing the differences between chiral and achiral compounds.

\section{Results and Discussion}

The main DPP core was synthesized by the cyclization reaction between 2-thiophenecarbonitrile and diethylsuccinate, following a reported protocol.[44] The final DPPBA, (S)-DPPBA and (R)-DPPBA were obtained via alkylation reactions with the mesylated amide-containing branches (Scheme 1). These branches were synthesized by reacting $\varepsilon$-caprolactone with the corresponding amine derivatives (chiral and achiral) and subsequently adding the mesylate activating group (Scheme 1). The DPP was N-alkylated with cesium carbonate as the base to deprotonate and $\mathrm{N}, \mathrm{N}$-dimethylformamide (DMF) as the solvent as in similar DPP alkylation reactions reported. $[45,46]$ The yields are low $(10 \%)$ because of the produced sub-products isolated and identified, such as the $\mathrm{O}$ alkylated isomers. Different reaction conditions were tested to increase the $\mathrm{N}$-alkylation yield. For instance, the reactions were performed under microwave irradiation as the heating source. In a first attempt, the reaction was carried out at room temperature to slow the formation of sub-products, since at high temperature and basic conditions, both $\mathrm{N}$ - and O-alkylation are favoured.[47] After one hour, only the mono $\mathrm{N}$-alkylated product was obtained together with unreacted DPP and mesylate. When the reaction time increased, the O-alkylated isomers was already form as soon as the desired product is formed with a low yield (5-8\%). In a second attempt, the reaction was performed at $12{ }^{\circ} \mathrm{C}$ for one hour, adding a few drops of DMF to make a heterogeneous reaction mixture, demonstrated to work better for microwave reactions[48,49]. In this case, the major products were the $\mathrm{N}$-alkylated derivatives accompanied by the O-alkylated isomer. Both products were isolated by column chromatography, finding yields of $15 \%$ for the (S)enantiomer, but not higher than $10 \%$ for the $(R)$. The reactions were performed at lower temperatures $(70-80 \stackrel{\circ}{\circ})$ to achieve the bisalkylated derivatives and try to avoid O-alkylated isomers. However, the (S)-enantiomer was obtained in $8 \%$ yield, and the (R) in yields of $15-22 \%$. In acetonitrile, a greener solvent, the yields were even lower either with microwave irradiation or conventional heating.

All the derivatives were characterized using proton and carbon nuclear magnetic resonance (NMR), and mass spectrometries. The final products were as well characterized with elemental analysis (See SI for further details). UV-Vis, FTIR and CD spectroscopy analyses were performed in solution and in the solid state to shed light into the assembly processes of the three derivatives, and transmission electron microscopy (TEM) was used to study the formation of supramolecular structures. Chloroform solutions (2.5 $\mathrm{mg} / \mathrm{mL}$ ) showed broad absorption bands ranging from 415 to $600 \mathrm{~nm}$ for the three derivatives at $20{ }^{\circ} \mathrm{C}$ (Figure $1 \mathrm{a}$ ). The chiral derivatives showed an additional absorption band at lower energy at $600 \mathrm{~nm}$ (Figure 1a, traces red and blue). This band was not observed for DPPBA, even at much higher concentration (Figure 1a). This band could be attributed to the formation of J-type aggregates, as for other H-bonded DPP derivatives, including semicarbazone-containing DPPs reported earlier by our group.[19,5052] This signal is related to the formation of $\mathrm{H}$-bonds since it disappears when methanol, an $\mathrm{H}$-bonding competing solvent, is added to (S)-DPPBA and (R)-DPPBA (Figure S1).

FTIR spectra of chloroform solutions at room temperature prove the existence of $\mathrm{H}$-bonds in the bisamide derivatives (Figure $2 \mathrm{a}$ ). DPPBA showed only one band at $3450 \mathrm{~cm}-1$ in the NH stretching area, which is characteristic of the $\mathrm{NH}$ stretching mode (amide A) of free amide groups. This result points out that DPPBA is not $\mathrm{H}$-bonded in chloroform. The amide I mode is found at $1659 \mathrm{~cm}-1$, which also corresponds to free amide groups. On the other hand, (S)-DPPBA and (R)-DPPBA showed two bands at $3439 \mathrm{~cm}-1$ and $3297 \mathrm{~cm}-1$ in the NH stretching area at room temperature (Figure 2a). The first band can be attributed to the free amide, analogously to the band at $3450 \mathrm{~cm}-1$ for DPPBA; the second band corresponds to $\mathrm{H}$-bonded amide groups. Hence, (S)-DPPBA and (R)-DPPBA are partially $\mathrm{H}$-bonded and part of it is free. In the region between $1600 \mathrm{~cm}-1$ and $1700 \mathrm{~cm}-1$, (S)-DPPBA and (R)-DPPBA show three peaks at $1632 \mathrm{~cm}-1,1648 \mathrm{~cm}-1$ and $1663 \mathrm{~cm}-1$. The peak at $1632 \mathrm{~cm}-1$ corresponds to amide groups engaged in strong $\mathrm{H}$ bonds[53], while the peak at $1648 \mathrm{~cm}-1$ corresponds to weaker bonds. The peak at $1663 \mathrm{~cm}-1$ matches with the peak for free amide observed with DPPBA. When increasing gradually the temperature from 27 to $59{ }^{\circ} \mathrm{C}$ the spectra of DPPBA did not show significant evolution. In contrast, for the chiral derivatives, the peaks corresponding to $\mathrm{H}$-bonded amides gradually decreased and fully disappear at $45^{\circ} \mathrm{C}$. At this temperature, the spectrum of (S)-DPPBA matches the one of DPPBA at room temperature (Figure S2).

The optical properties were measured in different solvents: toluene, chlorobenzene, cyclohexane and ethyl acetate. In these solvents, the J-aggregate band becomes more intense than in chloroform for the three derivatives (Figure $1 \mathrm{~b}-\mathrm{d}$ ). A shoulder band appears at approximately $480 \mathrm{~nm}$ in several solvents, attributed to the formation of $\mathrm{H}$-type aggregates. It is particularly intense in chlorobenzene, toluene and cyclohexane. In the case of (S)-DPPBA and (R)-DPPBA, the J-aggregate signal $(\square=600 \mathrm{~nm})$ is the predominant one in solvents like toluene, cyclohexane and ethyl acetate, showing the strong formation of $\mathrm{H}$-bonds in these conditions. In these solvents like in chloroform, the band disappears when methanol is added. (Figure S3a and S3b). Thanks to the formation of $\mathrm{J}$-aggregates it is possible to cover a larger zone of the spectrum and to tune the energy band gap. This is very interesting since the optoelectronic properties can be modified by varying the aggregation state without modifying the molecule. Furthermore, this strategy allows us, with very simple semiconducting segments, to control the properties through aggregation.

Dilution series and variable temperature measurements were performed to keep on exploring the aggregation process. The $\mathrm{J}$ aggregate bands were observed upon diluting, still appearing at low concentrations in solvents that promote the formation of strong 
$\mathrm{H}$-bonds (Figure S4). When the temperature increases, the J-aggregate band is still present, emphasizing the formation of aggregates even at high temperature (Figure S5a, b and c). This trend was also observed for the three derivatives, but the $\mathrm{J}$ aggregate bands disappeared at lower temperatures and higher concentrations for the chiral derivatives compared to the achiral one. This proves that the chiral derivatives form more stable aggregates than the achiral one. The UV-Vis spectra of the three derivatives in the solid state, were measured on cast thin films, showing a bathochromic shift with the appearance of a predominant $\mathrm{J}$-aggregate, as an effect of planarization. This result is independent from the casting solvent.(Figure S6).

CD spectra were measured in solution and showed signals with strong Cotton effect for the chiral derivatives, while no signal was observed for DPPBA even in aggregation conditions (Figure S7). Figure $2 b$ shows CD spectra in toluene for (S)-DPPBA and (R)DPPBA, where strong signals are found, especially at the J-aggregate wavelength. Variable temperature CD was performed in several solvents, finding strong signals at low temperature that gradually disappear upon heating. The CD signal can be observed up to high temperatures, reaching $60-80 \stackrel{\circ}{ }$, as evidenced in figure $2 \mathrm{c}$ and $2 \mathrm{~d}$, where the variable temperature spectra in chlorobenzene and toluene were recorded, respectively.

Morphology studies were performed using transmission electron microscopy (TEM) by depositing solutions of the amide-based DPPs in the solvents used for UV-visible and CD spectroscopy. Different long aspect-ratio structures were observed for the three derivatives in each solvent. The chiral derivatives show helical structures in most of the solvents, while the DPPBA shows different one-dimensional structures. Thin films of DPPBA in chloroform showed the presence of fibres with an average length of $10 \mu \mathrm{m}$ (Figure S9). In chlorobenzene and toluene, shorter structures were observed with an average length of $2 \mu \mathrm{m}$ that regroup in ribbonlike structures with widths of several hundreds of nanometers. Stiff fibres were observed in ethyl acetate and cyclohexane samples (Figure 3a-d). Regarding (S)-DPPBA (Figure 3e-h) and (R)-DPPBA (Figure 3i-l) samples, twisted fibres and helical structures were found in all the solvents studied, being more flexible than the structures of DPPBA, which seem stiffer (Figure 3). Nevertheless, less flexible structures were found for (R)-DPPBA and (S)-DPPBA in solvents like cyclohexane (Figure 3) and ethyl acetate (Figure S10). The photoconductivity of (S)-DPPBA and (R)-DPPBA self-assembled structures, as well as in DPPBA as a reference, were measured using FP-TRMC upon exposure to a $355 \mathrm{~nm}$ laser. FP-TRMC is an electrodeless technique that provides a measure of photoconductivity as $\Phi \Sigma \mu$ were $\Phi$ is the charge carrier generation quantum yield and $\Sigma \mu$ is the sum of charge carrier mobilities of electrons and holes.[54-56] Thin films of the compounds were prepared by drop casting from chloroform solutions, and their photoconductivity was analysed before and after solvent vapour annealing. The solvent annealing protocol was 1) to expose to ethyl acetate for $24 \mathrm{~h}$, and 2) to methanol vapour for $24 \mathrm{~h}$ : ethyl acetate promotes efficiently aggregation of the molecules, and methanol disrupts $\mathrm{H}$-bonds. The observed kinetic traces of conductivity transients are shown on figure 4 . The photoconductivity of DPPBA is evidently larger than those of (S)- and (R)-DPPBA (the values can be found in table S1), even though photoconductivity $(\Phi \Sigma \mu)$ depends on the photo generation yield $\Phi$. The kinetics decay of photoconductivity of DPPBA shows that the photo-generated free charge carriers are stable up to $10 \mu \mathrm{s}$. The recombination of charge carriers is slower in DPPBA than in (S)- and (R)-DPPBA, with one order of magnitude longer lifetime, being $t_{1 / 2}$ of DPPBA $2.1 \times 10^{-5} \mathrm{~s}$ and $4.5 \times 10^{-6} \mathrm{~s}$ and $6.6 \times 10^{-6} \mathrm{~s}$ for (S)-DPPBA and (R)DPPBA, respectively (Table S2). It seems that the lower solubility of the chiral DPPs and the presence of the methyl group next to the amide group are detrimental for the charge carrier pathways.

To assess the impact of the self-assembled structure quantitatively on the charge carrier pathways, the photoconductivity signal was traced at each step of the solvent annealing protocol in an identical film of each compound. Interestingly, the highest photoconductivity $\left(7.5 \times 10^{-5} \mathrm{~cm}^{2} \mathrm{~V}^{-1} \mathrm{~s}^{-1}\right.$, table S1) was recorded in DPPBA after solvent annealing in ethyl acetate (Figure 4d), suggesting an efficient promotion of aggregated states of the molecule. The photoconductivity value increased up to 1.5 fold from the initial $5.0 \times 10^{-5} \mathrm{~cm}^{2} \mathrm{~V}^{-1} \mathrm{~s}^{-1}$, emphasizing the importance of promoting the $\mathrm{H}$-bonding strength. On the other hand, methanol vapour annealing did not show any significant effect on photoconductivity (Figure 4e) in DPPBA. It seems that only methanol vapour is not enough to disrupt $\mathrm{H}$-bonds in the condensed phase. However, when the film previously exposed to ethyl acetate was soaked in methanol, the photoconductivity decreased dramatically as well as the lifetime (Figure 4f, Table S3). This result highlights the important role of $\mathrm{H}$-bonding to facilitate the charge carrier pathways in the self-assembled structures. Regarding the chiral derivatives (S)- and (R)-DPPBA, no differences in photoconductivity were found during the solvent annealing protocols (Figure S11, Table S1). This would suggest that tightly-bound self-assembled structures were already formed in the solution phases, and the annealing is no longer efficient to cause significant rearrangements in the molecular packing structures of chiral substances. The presence of a methyl group next to the $\mathrm{H}$-bonding (amide) motif and the lower solubility of both chiral isomers compared to DPPBA, might be supportive for the stability of the self-assembled structures.

\section{Conclusion}

In conclusion, we have synthesized and characterized three amide-containing DPP derivatives, two of them bearing chiral centers. The three derivatives aggregate and form J-type aggregates, where $\mathrm{H}$-bonds are involved. The aggregation is particularly strong in the chiral derivatives, which show aggregate signals in solvents where the achiral DPP is fully dissociated. The photoconductivity measurements performed using FP-TRMC indicate that the achiral derivative has superior values of photoconductivity as well as slower recombination of the charge carriers. The importance of $\mathrm{H}$-bonding is highlighted by annealing samples in solvents that promote self-assembly, where the photoconductivity values increased. In contrast, the photoconductivity values decreased dramatically when the samples were soaked in H-bonding competing solvents. This effect was not observed for the chiral derivatives, indicating that the chiral center next to the $\mathrm{H}$-bonding functionality might hinder charge transport. Our future efforts are focused on studying different chiral centers and the optimal distance to the $\mathrm{H}$-bonding motif. 


\section{Experimental Section}

All reagents and solvents were obtained from commercial suppliers and purified or dried according to standard procedures. Column chromatography was performed on silica gel (VWR Silica 60, particle size $0.040-0.063 \mathrm{~mm}$ ). Solvents for spectroscopic studies were of spectroscopic grade and used as received. Elemental analysis was performed on a Thermofischer Scientific Flash 2000. Matrix-assisted laser desorption/ionisation-time of filgh (MALDI) was performed in a Bruker Daltonics. ${ }^{1} \mathrm{H}$ and ${ }^{13} \mathrm{C}$ spectra were recorded in $\mathrm{CDCl}_{3}$ on a Bruker Avance $400 \mathrm{MHz}$ spectrometer and/or Bruker Avance III HD $500 \mathrm{MHz}$ spectrometer. UV-vis measurements were performed in a conventional quartz cell (light pass $1 \mathrm{~mm}$ ) on a Cary 5000 UV-VisNIR spectrophotometer. TEM measurements were done with a Technai G2 (FEI) microscope with an accelerating voltage of $200 \mathrm{kV}$. $5 \mu \mathrm{L}$ of the sample solution were deposited onto a freshly glow discharged carbon-covered grid (400 mesh). The suspension was left for 2 min, and then, the grid was negatively stained with $5 \mu \mathrm{L}$ of uranyl acetate ( $2 \%$ in water) for another $1 \mathrm{~min}$ and finally blotted using a filter paper.

FTIR spectra were recorded with a Vertex 70 from Bruker Optics, equipped with MCT detector and a black-body source. The spectra of the solids were measured by ATR on diamond. The solutions were studied in cells from Specac Pike with $\mathrm{KBr} \mathrm{NaCl}$ windows. For the VT experiments the solutions were inserted in a home-made cell between two $\mathrm{NaCl}$ windows. The cell was inserted in a Linkam heating stage, hold in a horizontal measurement chamber. The spectra were measured with the built-in MCT detector of the Vertex 70 . The spectra were compensated from $\mathrm{CO}_{2}$ and moisture with OPUS from Bruker. The solvent intensities were measured separately and subtracted. The spin coated samples were prepared using a Spin 150 spin coater at $2000 \mathrm{rpm}$ using $80 \mu \mathrm{l}$ of solution on glass slides or silicon wafers.

Charge carrier mobilities were evaluated by FP-TRMC at room temperature. ${ }^{56,57}$ The thin films of samples were fabricated by drop-cast method onto quartz substrates $\left(9 \times 40 \mathrm{~mm}^{2}, 1 \mathrm{~mm}\right.$ thick). Charge carriers were injected into the materials via photo-ionization with a third harmonic generation $(\lambda=$ $355 \mathrm{~nm}$ ) of a Spectra Physics model INDI-HG Nd:YAG laser pulses at $10 \mathrm{~Hz}$ with a pulse duration of ca. $5 \mathrm{~ns}$. The photon density of a $355 \mathrm{~nm}$ pulse was modulated from $4.5 \times 10^{15}$ photons $\mathrm{cm}^{-2}$ pulse ${ }^{-1}$. The microwave frequency and power were set at $\sim 9.1 \mathrm{GHz}$ and $3 \mathrm{~mW}$, respectively, and guided into a microwave cavity. The $Q$-factor of the microwave cavity loaded with the sample was 2200 , and the substrates with the compound films were set at the point of electric field maximum. The reflected power of the probing microwave, picked up by a diode (rise time $<1$ ns), was monitored by a Tektronics model TDS3032B digital oscilloscope after an amplification by Ciao Electronics CA812-304 FET amplifier system. The observed change in the reflected microwave power $\left(\Delta P_{\mathrm{r}}\right)$ was normalised with the steady reflection of the microwave from the cavity $\left(P_{\mathrm{r}}\right)$, and converted directly into the product of a photocarrier generation yield $(\phi)$ and the sum of photo-generated electron/hole mobilities $(\Sigma \mu)$,

$\phi \Sigma \mu=1 /\left(\varepsilon l_{0} F_{\text {light }}\right) \times(1 / A) \times\left(\Delta P_{\mathrm{r}} / P_{\mathrm{r}}\right)$

where $\varepsilon, A, I_{0}$, and $F_{\text {light }}$ are elementary charge, sensitivity factor $\left(\mathrm{S}^{-1} \mathrm{~cm}\right)$, incident photon density of the excitation laser (photon $\mathrm{cm}^{-2}$ ), and filling factor $\left(\mathrm{cm}^{-1}\right)$, respectively. The value of Flight was calculated based on the overlap of the area of photo-carrier injection (presumed to be proportional to the absorbance of excitation light by the sample film) with electric field strength distribution in the cavity derived from a calculation code of CST Microwave Studio from AET Inc.

\section{Acknowledgements}

The authors acknowledge the characterization facility for UV-Vis and FTIR and the microscopy platform for the use of SEM of Insitut Charles Sadron (CNRS). The authors thank Christian Blanck and Marc Schmutz for the TEM imaging A. R. C. and N. R. A. R. thank the Foundation for Frontier Research in Chemistry (FRC) LabEx Emerging Investigators Grant 2018 and CNRS for financial support. S. M., P. J. M. and A. R. C. thank the International Research Training Center (IRTG) SoMas and the Région Grand-Est for the doctoral fellowship of S. M. The authors gratefully acknowledge Jean-Philippe Lamps for laboratory technical support.

Keywords: hydrogen-bonding $\bullet$ self-assembly $\bullet$ supramolecular chirality $\bullet$ diketopyrrolopyrrole $\bullet$ flash photolysis time resolved microwave conductivity

1. Facchetti, A. (2011) $\pi$-Conjugated Polymers for Organic Electronics and Photovoltaic Cell Applications. Chem. Mater., 23 (3), $733-758$.

2. Mishra, A., and Bäuerle, P. (2012) Small Molecule Organic Semiconductors on the Move: Promises for Future Solar Energy Technology. Angew. Chem. Int. Ed., 51 (9), 2020-2067.

3. McDearmon, B., Page, Z.A., Chabinyc, M.L., and Hawker, C.J. (2018) Organic electronics by design: the power of minor atomic and structural changes. J. Mater. Chem. C, 6 (14), 3564-3572.

4. Poelking, C.R. (2018) Organic Electronics in a Nutshell, in The (Non-)Local Density of States of Electronic Excitations in Organic Semiconductors, Springer, Cham, pp. 1-11.

5. Choi, D., Chu, P.-H., McBride, M., and Reichmanis, E. (2015) Best Practices for Reporting Organic Field Effect Transistor Device Performance. Chem. Mater., 27 (12), 4167-4168.

6. Thejo Kalyani, N., and Dhoble, S.J. (2012) Organic light emitting diodes: Energy saving lighting technology—A review. Renew. Sustain. Energy Rev., 16 (5), 2696-2723.

7. Hoppea, H., and Sariciftci, N.S. (2004) Organic solar cells: An overview. J Mater Res, 19 (7), 1925.

8. Russ, B., Glaudell, A., Urban, J.J., Chabinyc, M.L., and Segalman, R.A. (2016) Organic thermoelectric materials for energy harvesting and temperature control. Nat. Rev. Mater., 1 (10), 16050. 
9. Baert, F., Cabanetos, C., Allain, M., Silvestre, V., Leriche, P., and Blanchard, P. (2016) Thieno[2,3-b]indole-Based Small Push-Pull Chromophores: Synthesis, Structure, and Electronic Properties. Org. Lett., 18 (7), 1582-1585.

10. Hutchison, G.R., Ratner, M.A., and Marks, T.J. (2005) Intermolecular charge transfer between heterocyclic oligomers. Effects of heteroatom and molecular packing on hopping transport in organic semiconductors. J Am Chem Soc, 127 (48), 16866-16881.

11. M. Randell, N., C. Boutin, P., and L. Kelly, T. (2016) Bisisoindigo: using a ring-fusion approach to extend the conjugation length of isoindigo. J. Mater. Chem. A, 4 (18), 6940-6945.

12. Lee, O.P., Yiu, A.T., Beaujuge, P.M., Woo, C.H., Holcombe, T.W., Millstone, J.E., Douglas, J.D., Chen, M.S., and Fréchet, J.M.J. (2011) Efficient Small Molecule Bulk Heterojunction Solar Cells with High Fill Factors via Pyrene-Directed Molecular Self-Assembly. Adv. Mater., 23 (45), 5359-5363.

13. Verploegen, E., Mondal, R., Bettinger, C.J., Sok, S., Toney, M.F., and Bao, Z. (2010) Effects of Thermal Annealing Upon the Morphology of Polymer-Fullerene Blends. Adv. Funct. Mater., 20 (20), 3519-3529.

14. Conboy, J.C., Olson, E.J., Adams, D.M., Kerimo, J., Zaban, A., Gregg, B.A., and Barbara, P.F. (1998) Impact of solvent vapor annealing on the morphology and photophysics of molecular semiconductor thin films. J. Phys. Chem. B, 102 (23), $4516-4525$.

15. Biniek Laure, Hamidi-Sakr Amer, Grodd Linda, Escoubas Stéphanie, Dappe Yannick J., Grigorian Souren, Schmutz Marc, and Brinkmann Martin (2018) Structure and Charge Transport Anisotropy of Polythieno[3,4-b]-Thiophene-co-Benzodithiophene (PTB7) Oriented by High-Temperature Rubbing. Adv. Electron. Mater., 0 (0), 1700480.

16. Steinberger, S., Mishra, A., Reinold, E., Levichkov, J., Uhrich, C., Pfeiffer, M., and Bäuerle, P. (2011) Vacuum-processed small molecule solar cells based on terminal acceptor-substituted low-band gap oligothiophenes. Chem. Commun., 47 (7), 1982.

17. Punzi, A., Operamolla, A., Hassan Omar, O., Brunetti, F., Scaccabarozzi, A.D., Farinola, G.M., and Stingelin, N. (2018) Designing Small Molecules as Ternary Energy-Cascade Additives for Polymer:Fullerene Solar Cell Blends. Chem. Mater., 30 (7), $2213-2217$.

18. Aida, T., Meijer, E.W., and Stupp, S.I. (2012) Functional supramolecular polymers. Science, 335 (6070), $813-817$.

19. Militzer, S., Tran, T.M.P., Mésini, P.J., and Ruiz-Carretero, A. (2018) Tuning the Optical and Self-Assembly Properties of Diketopyrrolopyrrole Semicarbazone Derivatives through Hydrogen Bonding. ChemNanoMat, 4 (8), $790-795$.

20. Tsai, Y.-T., Tseng, K.-P., Chen, Y.-F., Wu, C.-C., Fan, G.-L., Wong, K.-T., Wantz, G., Hirsch, L., Raffy, G., Del Guerzo, A., and Bassani, D.M. (2016) Electroluminescence from Spontaneously Generated Single-Vesicle Aggregates Using Solution-Processed Small Organic Molecules. ACS Nano, 10 (1), 998-1006.

21. Velu, S.K.P., Yan, M., Tseng, K.-P., Wong, K.-T., Bassani, D.M., and Terech, P. (2013) Spontaneous Formation of Artificial Vesicles in Organic Media through Hydrogen-Bonding Interactions. Macromolecules, 46 (4), 1591-1598.

22. Ghosh, G., Paul, M., Sakurai, T., Matsuda, W., Seki, S., and Ghosh, S. (2018) Supramolecular Chirality Issues in Unorthodox Naphthalene Diimide Gelators. Chem. - Eur. J., 24 (8), 1938-1946.

23. Supramolecular Chirality in Self-Assembled Systems - Chemical Reviews (ACS Publications).

24. Shang, X., Song, I., Ohtsu, H., Lee, Y.H., Zhao, T., Kojima, T., Jung, J.H., Kawano, M., and Oh, J.H. (2017) Supramolecular Nanostructures of Chiral Perylene Diimides with Amplified Chirality for High-Performance Chiroptical Sensing. Adv. Mater., 29 (21), 1605828.

25. Schenning, A.P.H.J., Jonkheijm, P., Peeters, E., and Meijer, E.W. (2001) Hierarchical Order in Supramolecular Assemblies of HydrogenBonded Oligo(p-phenylene vinylene)s. J. Am. Chem. Soc., 123 (3), 409-416.

26. Würthner, F., Chen, Z., Hoeben, F.J., Osswald, P., You, C.-C., Jonkheijm, P., Herrikhuyzen, J. v, Schenning, A.P., van der Schoot, P.P., and Meijer, E.W. (2004) Supramolecular p-n-Heterojunctions by Co-Self-Organization of Oligo (p-phenylene Vinylene) and Perylene Bisimide Dyes. J. Am. Chem. Soc., 126 (34), 10611-10618.

27. Ruiz-Carretero, A., Rovelo, N.R.Á., Militzer, S., and Mésini, P.J. (2019) Hydrogen-bonded diketopyrrolopyrrole derivatives for energy-related applications. J. Mater. Chem. A, 7 (41), 23451-23475.

28. Grzybowski, M., and Gryko, D.T. (2015) Diketopyrrolopyrroles: Synthesis, Reactivity, and Optical Properties. Adv. Opt. Mater., 3 (3), 280320.

29. Tang, A., Zhan, C., Yao, J., and Zhou, E. (2017) Design of Diketopyrrolopyrrole (DPP)-Based Small Molecules for Organic-Solar-Cell Applications. Adv. Mater., 29 (2), 1600013.

30. Li, W., Hendriks, K.H., Wienk, M.M., and Janssen, R.A.J. (2016) Diketopyrrolopyrrole Polymers for Organic Solar Cells. Acc. Chem. Res., 49 (1), 78-85.

31. Yang, X., Zheng, L., Xie, L., Liu, Z., Li, Y., Ning, R., Zhang, G., Gong, X., Gao, B., Liu, C., Cui, Y., Sun, G., and Zhang, G. (2015) Colorimetric and On-Off fluorescent chemosensor for fluoride ion based on diketopyrrolopyrrole. Sens. Actuators B Chem., $207,9-24$. 
32. Bürckstümmer, H., Weissenstein, A., Bialas, D., and Würthner, F. (2011) Synthesis and Characterization of Optical and Redox Properties of Bithiophene-Functionalized Diketopyrrolopyrrole Chromophores. J. Org. Chem., 76 (8), 2426-2432.

33. Liu, J., Zhang, Y., Phan, H., Sharenko, A., Moonsin, P., Walker, B., Promarak, V., and Nguyen, T.-Q. (2013) Effects of Stereoisomerism on the Crystallization Behavior and Optoelectrical Properties of Conjugated Molecules. Adv. Mater., 25 (27), 3645-3650.

34. Zerdan, R.B., Shewmon, N.T., Zhu, Y., Mudrick, J.P., Chesney, K.J., Xue, J., and Castellano, R.K. (2014) The Influence of Solubilizing Chain Stereochemistry on Small Molecule Photovoltaics. Adv. Funct. Mater., 24 (38), 5993-6004.

35. He, T., Leowanawat, P., Burschka, C., Stepanenko, V., Stolte, M., and Würthner, F. (2018) Impact of 2-Ethylhexyl Stereoisomers on the Electrical Performance of Single-Crystal Field-Effect Transistors. Adv. Mater., 30 (44), 1804032

36. Stolte, M., Suraru, S.-L., Diemer, P., He, T., Burschka, C., Zschieschang, U., Klauk, H., and Wurthner, F. (2016) Diketopyrrolopyrrole Organic Thin-Film Transistors: Impact of Alkyl Substituents and Tolerance of Ethylhexyl Stereoisomers. Adv Mater, 26 (41), $7415-7422$.

37. Dhbaibi, K., Favereau, L., Srebro-Hooper, M., Jean, M., Vanthuyne, N., Zinna, F., Jamoussi, B., Bari, L.D., Autschbach, J., and Crassous, J. (2018) Exciton coupling in diketopyrrolopyrrole-helicene derivatives leads to red and near-infrared circularly polarized luminescence. Chem. Sci., 9 (3), $735-742$.

38. Hume, P.A., Monks, J.P., Pop, F., Davies, E.S., MacKenzie, R.C.I., and Amabilino, D.B. Self-Assembly of Chiral-at-End Diketopyrrolopyrroles: Symmetry Dependent Solution and Film Optical Activity and Photovoltaic Performance. Chem. - Eur. J., 0 (0).

39. Rieth, S., Li, Z., Hinkle, C.E., Guzman, C.X., Lee, J.J., Nehme, S.I., and Braunschweig, A.B. (2013) Superstructures of Diketopyrrolopyrrole Donors and Perylenediimide Acceptors Formed by Hydrogen-Bonding and $\pi \cdots \pi$ Stacking. J. Phys. Chem. C, 117 (21), 11347-11356.

40. Zhou, Y., Guzman, C.X., Helguero-Kelley, L.C., Liu, C., Peurifoy, S.R., Captain, B., and Braunschweig, A.B. (2016) Diketopyrrolopyrrole assembly into J-aggregates. J. Phys. Org. Chem., 29 (12), 689-699.

41. Guzman, C.X., Calderon, R.M.K., Li, Z., Yamazaki, S., Peurifoy, S.R., Guo, C., Davidowski, S.K., Mazza, M.M.A., Han, X., Holland, G., Scott, A.M., and Braunschweig, A.B. (2015) Extended Charge Carrier Lifetimes in Hierarchical Donor-Acceptor Supramolecular Polymer Films. J. Phys. Chem. C, 119 (34), 19584-19589.

42. Ley, D., Guzman, C.X., Adolfsson, K.H., Scott, A.M., and Braunschweig, A.B. (2014) Cooperatively Assembling Donor-Acceptor Superstructures Direct Energy Into an Emergent Charge Separated State. J. Am. Chem. Soc., 136 (22), $7809-7812$.

43. Peurifoy, S.R., Guzman, C.X., and Braunschweig, A.B. (2015) Topology, assembly, and electronics: three pillars for designing supramolecular polymers with emergent optoelectronic behavior. Polym. Chem., 6 (31), 5529-5539.

44. Iqbal, A., Jost, M., Kirchmayr, R., Pfenninger, J., Rochat, A., and Wallquist, O. (1988) The synthesis and properties of 1, 4-diketo-pyrrolo [3, 4-C] pyrroles. Bull. Soc. Chim. Belg., 97 (8-9), 615-644.

45. Ruiz-Carretero, A., Aytun, T., Bruns, C.J., Newcomb, C.J., Tsai, W.-W., and Stupp, S.I. (2013) Stepwise self-assembly to improve solar cell morphology. J. Mater. Chem. A, 1 (38), 11674.

46. Loser, S., Bruns, C.J., Miyauchi, H., Ortiz, R.P., Facchetti, A., Stupp, S.I., and Marks, T.J. (2011) A NaphthodithiopheneDiketopyrrolopyrrole Donor Molecule for Efficient Solution-Processed Solar Cells. J. Am. Chem. Soc., 133 (21), $8142-8145$.

47. Pop, F., Humphreys, J., Schwarz, J., Brown, L., Berg, A. van den, and Amabilino, D.B. (2019) Towards more sustainable synthesis of diketopyrrolopyrroles. New J. Chem., 43 (15), 5783-5790.

48. Ruiz-Carretero, A., Ramírez, J.R., Sánchez-Migallón, A., and de la Hoz, A. (2014) Microwave-assisted selective and efficient synthesis of 1,3,5-triazinyl mono and bisureas. Tetrahedron, 70 (9), 1733-1739.

49. Dressen, M.H.C.L., van de Kruijs, B.H.P., Meuldijk, J., Vekemans, J.A.J.M., and Hulshof, L.A. (2011) Variable Microwave Effects in the Synthesis of Ureidopyrimidinones: the Role of Heterogeneity. Org. Process Res. Dev., 15 (1), 140-147.

50. Mizuguchi, J., and Rihs, G. (1992) Electronic Spectra of 1,4-Diketo-3,6-Diphenyl-Pyrrolo-[3,4-c]-Pyrrole in the Solid State. Berichte Bunsenges. Für Phys. Chem., 96 (4), 597-606.

51. Mizuguchi, J. (1993) Solution and Solid State Properties of 1,4-Diketo-3,6-bis-(4'-pyridyl)-pyrrolo-[3,4-c]-pyrrole on Protonation and Deprotonation. Berichte Bunsenges. Für Phys. Chem., 97 (5), 684-693.

52. Ghosh, S., Cherumukkil, S., Suresh, C.H., and Ajayaghosh, A. (2017) A Supramolecular Nanocomposite as a Near-Infrared-Transmitting Optical Filter for Security and Forensic Applications. Adv. Mater., 29 (46), 1703783.

53. Eaton, G., Symons, M.C.R., and Rastogi, P.P. (1989) Spectroscopic studies of the solvation of amides with N-H groups. Part 1.-The carbonyl group. J. Chem. Soc. Faraday Trans. 1 Phys. Chem. Condens. Phases, 85 (10), 3257-3271.

54. Ghosh, S., Raveendran, R., Saeki, A., Seki, S., Namboothiry, M., and Ajayaghosh, A. (2019) Charge Carrier Polarity Modulation in Diketopyrrolopyrrole-Based Low Band Gap Semiconductors by Terminal Functionalization. ACS Appl. Mater. Interfaces, 11 (1), $1088-1095$. 
55. Matsuda, W., Sakurai, T., Ghosh, G., Ghosh, S., and Seki, S. (2018) Transient Optical-Microwave Spectroscopy for Electron Mobility Assessment in Solids and Gels: A Comprehensive Approach. J. Photopolym. Sci. Technol., 31 (1), 91-99.

56. Tsutsui, Y., Okamoto, H., Sakamaki, D., Sugiyasu, K., Takeuchi, M., and Seki, S. (2018) Landscape of Charge Carrier Transport in Doped Poly(3-hexylthiophene): Noncontact Approach Using Ternary Combined Dielectric, Paramagnetic, and Optical Spectroscopies. J. Phys. Chem. Lett., 9 (13), 3639-3645.

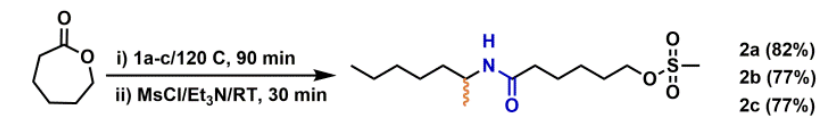<smiles>CCCCCCCCC(C)N</smiles><smiles>O=C1NC(c2cccs2)=C(c2cccs2)C1=O</smiles>
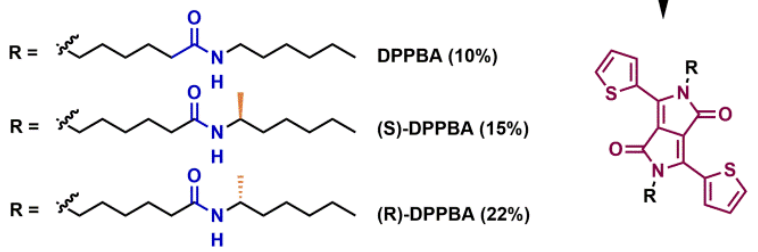

Scheme 1. Synthetic pathway towards DPPBA, (S)-DPPBA and (R)-DPPBA. iii) Microwave irradiation $(\mathrm{P}=150 \mathrm{~W}, \mathrm{DMF}, 2 \mathrm{~h}, 120 \stackrel{\circ}{\circ} \mathrm{C}$ for (S)-DPPBA and P = 150 W, DMF, $2 \mathrm{~h}, 70^{\circ} \mathrm{C}$ for (R)-DPPBA).

a)

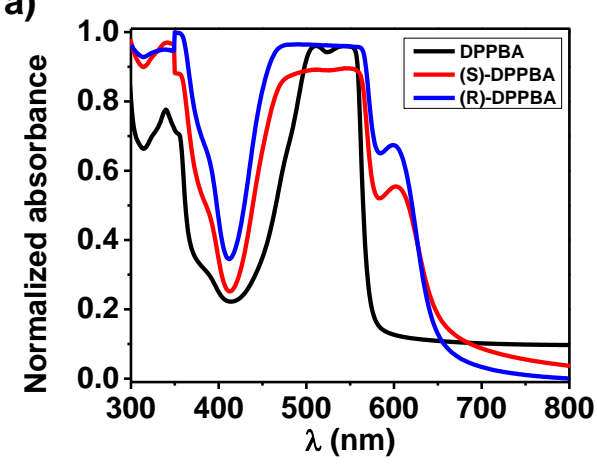

c)

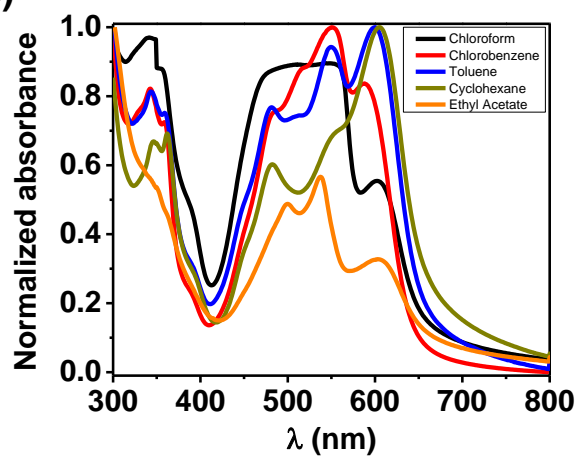

b)

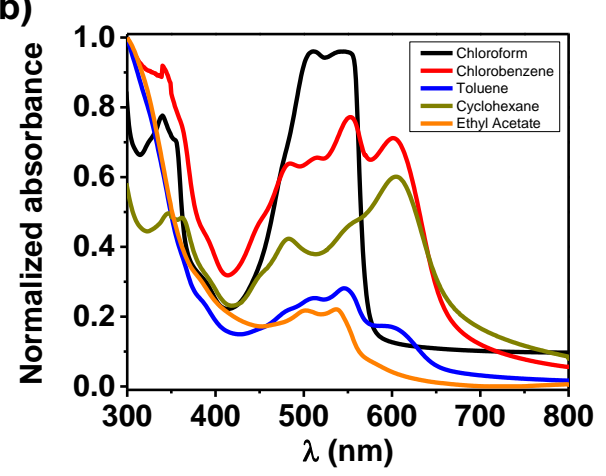

d)

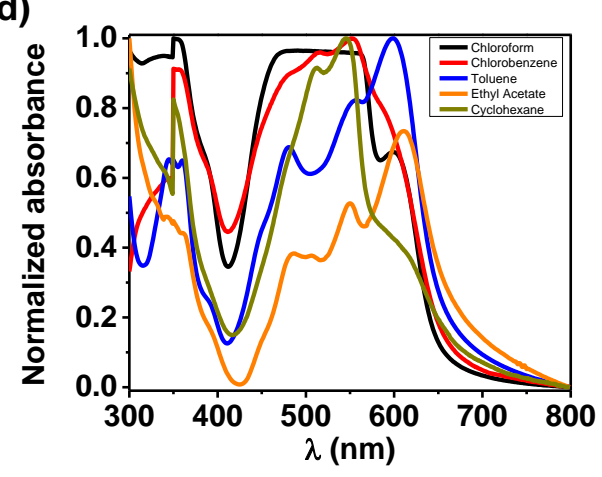

Figure 2. a) FTIR spectra in deuterated chloroform at room temperature of (S)-DPPBA, (R)-DPPBA and DPPBA. b) CD spectra (S)-DPPBA and (R)-DPPBA in toluene solution. [(S)-DPPBA ] $=[(\mathbf{R})$-DPPBA $]=2.5 \mathrm{mg} / \mathrm{ml}$. c) Variable temperature CD spectra of (S)-DPPBA in chlorobenzene. [(S)-DPPBA ] $=2.5 \mathrm{mg} / \mathrm{ml}$. d) $\begin{array}{llllll}\text { Variable temperature } \mathrm{CD} \text { spectra of } & \text { (R)-DPPBA in toluene. } \\ \text { [(R)-DPPBA] }\end{array}$ 

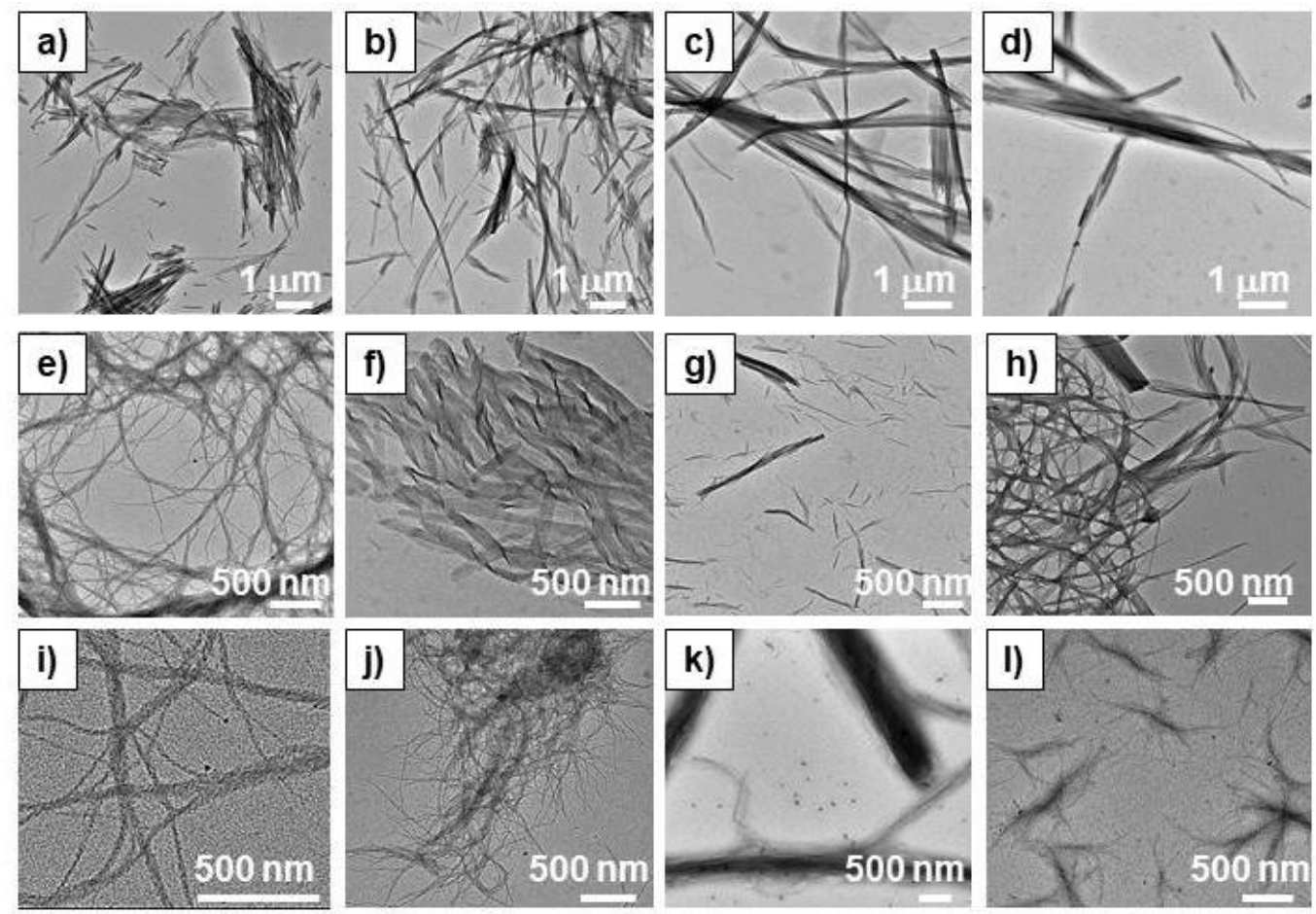

Figure 3. TEM images of DPPBA (a-d), (S)-DPPBA (e-h) and (R)-DPPBA (i-l) in chlorobenzene, toluene, cyclohexane and ethyl acetate, respectively.

a)

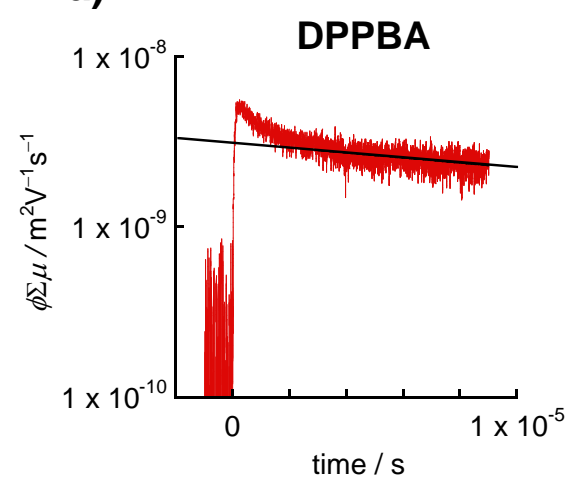

d)

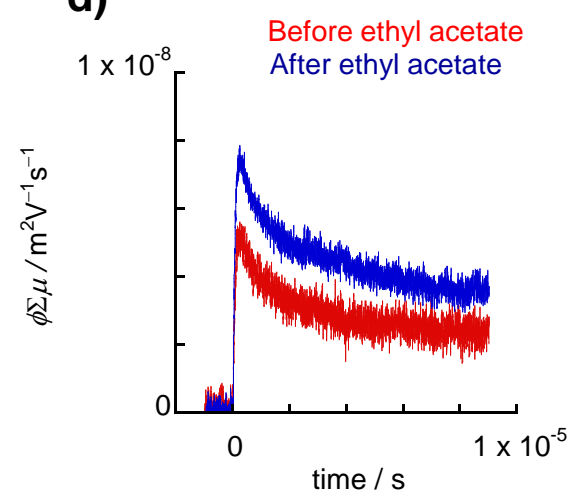

b)



e)

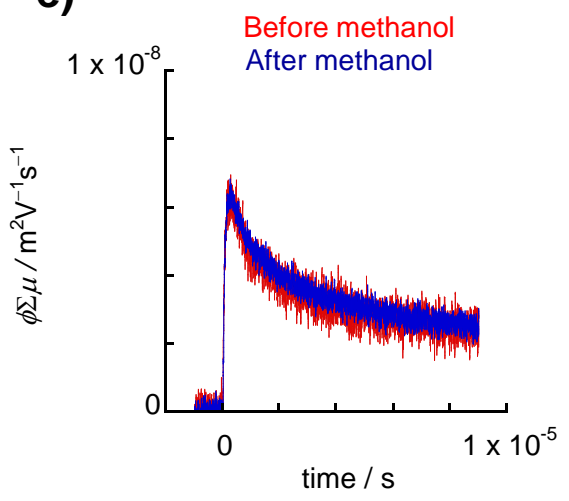

c)



f)

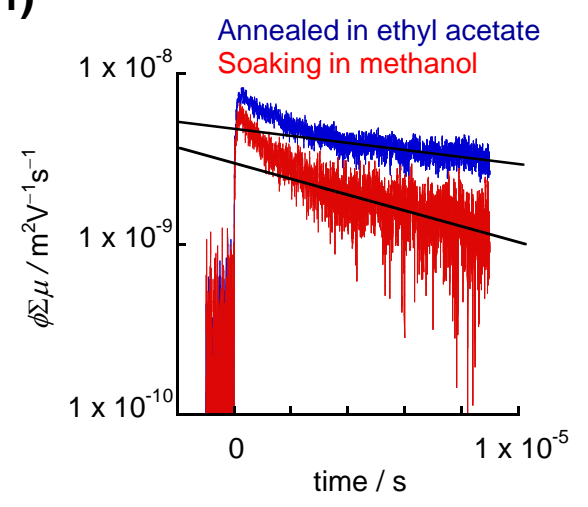

a)

b)

c) 